\title{
Frequency analysis of experimental waveforms for DC motors in digging applications
}

\author{
Galina Mirzaeva* $^{*}$ J.S. Welsh ${ }^{\star}$, T.J. Summers ${ }^{\dagger}$, R.E.Betz ${ }^{\ddagger}$ \\ School of Electrical Engineering and Computer Science \\ University of Newcastle, Australia, 2308 \\ email:*Galina.Mirzaeva@newcastle.edu.au; \\ *James.Welsh@newcastle.edu.au; \\ †Terry.Summers@newcastle.edu.au; \\ ${ }^{\ddagger}$ Robert.Betz@newcastle.edu.au
}

\begin{abstract}
This paper refers to a "DC Motor Duty Meter" project which aims to develop a comprehensive condition monitoring tool for DC motors used in mining excavators. The paper presents experimental waveforms measured internally and externally to the motor at different rotation velocities. The paper focuses on analysis of these waveforms both in time and frequency domains. The findings of the analysis are discussed in relation to the motor performance and commutation quality.
\end{abstract}

\section{INTRODUCTION}

DC motors are extensively used in open cut mining with digging machines such as electric rope shovels and draglines. The primary use of these machines is to remove blasted overburden (with a big proportion of hard rock of varying size) to expose a coal seam. Although a general tendency is to move towards $\mathrm{AC}$ machines, it is reasonable to expect that in digging applications DC machines will continue to be in service for many years in future. In such applications they are subject to very harsh conditions related to both physical environment and operational stresses. High ambient temperature, low humidity, high dust content, dynamically changing digging forces and shocks due to poorly fragmented rock pieces are some examples of such harsh conditions. At the same time, production needs call for increasingly bigger bucket sizes (i.e. increased rms loading) and shorter digging cycles (i.e. faster load dynamics) .

To ensure high reliability and availability of the motor fleet under the extreme conditions of their operation it is important to thoroughly understand different aspects of their performance. Furthermore, development of a condition monitoring tool for DC motors specific to the mining application would be very beneficial for the industry, so that production downtime due to catastrophic failures or unnecessary maintenance could be avoided or significantly reduced. The authors of this paper are currently involved in a research project supported by industry partners, which included construction of a test facility for full size (around 2000HP) industrial DC machines and an extensive experimental program under controlled steady state and dynamic conditions.

The structure of the facility was described in detail in [7] and is also briefly presented in a companion paper [1]. The facility is based on a back-to-back connection of two industrial

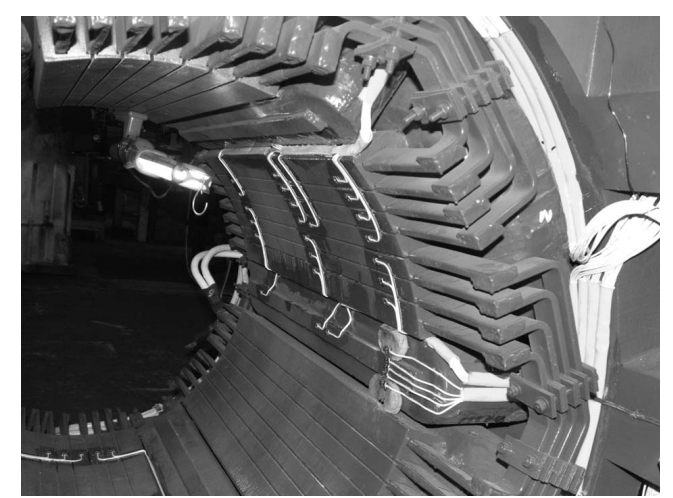

Figure 1. Sensors inside a DC motor

DC machines of the same size, each controlled by a digital drive with a thyristor rectifier (ABB DCS600 ${ }^{\circledR}$ ). Both machines are instrumented by a large number of sensors, which signals are recorded by a PC-based data acquisition system. Using an interface between the PCs and the digital drives, a dynamic dynamometer can be implemented as explained in [1]. This way one machine would represent the rope shovel DC motor (hoist or crowd), and the other machine would represent a dynamic load seen by the rope shovel motor under varying digging conditions.

The end goal of this project is to develop an on-line condition monitoring tool, initially, to monitor deterioration of the DC motor commutation group. The approach taken is based on a thorough understanding of the wear mechanisms, their mathematical modelling, and experimental verification of the models under physically simulated digging conditions. Finally, the on-line tool will be implemented that will relate continuously measured operating conditions to the rate of the brush and commutator wear and estimate their residual resource. One group of brush and commutator wear mechanisms is related to current density distributions between brushes and across each brush. This was investigated earlier and presented in [6]. The next area of interest, which is the focus of the current paper, is associated with the thyristor drive waveforms and their influence on the motor performance. Of particular 
a)

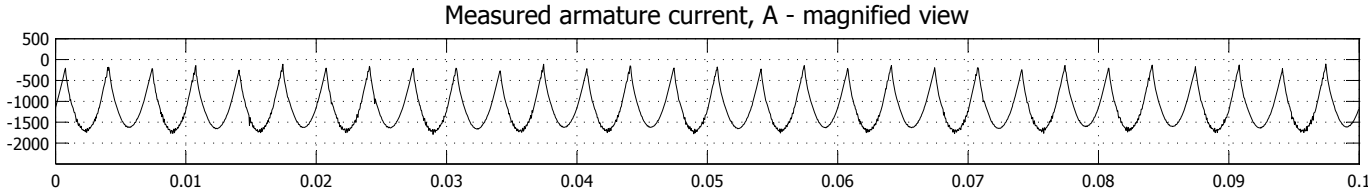

b)

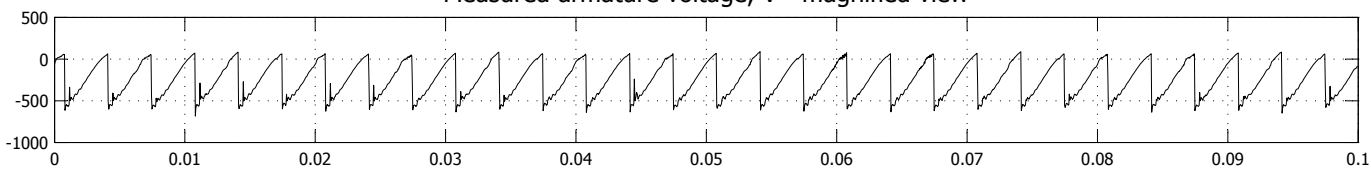

c)

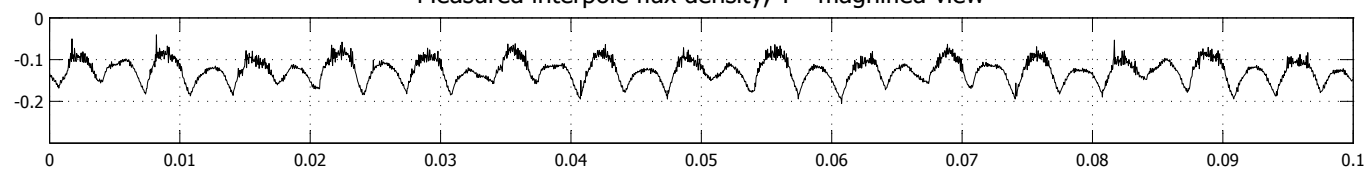

d)

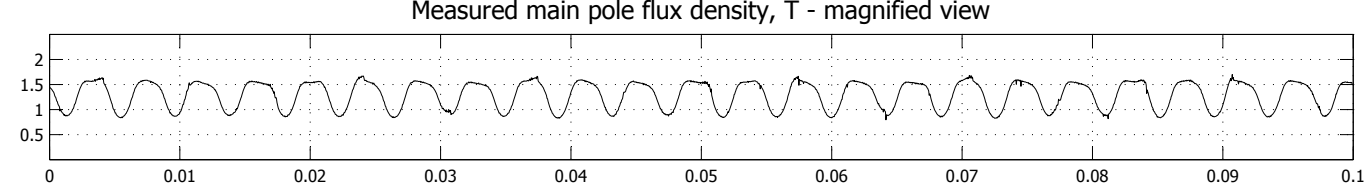

e)

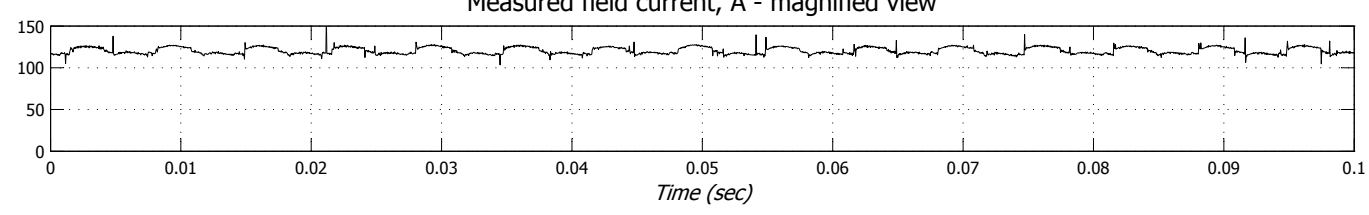

Figure 2. Fragment of experimental data at 243RPM and 1000A

interest are frequency components that may interfere with commutation or excite mechanical vibrations detrimental to the commutation group. Such components may be dependent on the rotational velocity of the motor, therefore, experimental data was collected under steady state conditions at different rotational velocities.

This paper is organised as follows. It first presents the experimental data and explains conditions under which they were obtained. Theory of the thyristor drive generated waveforms is then reviewed and extended to the motor internal signals (flux densities). The theory includes Fourier series representations, so that theoretical spectra of the signals of interest can be envisaged. Following this, results of the experimental data processing are presented and compared to the theoretically expected. The results are discussed with a particular emphasis on components that may be indicative of abnormal conditions. The attempt is then made to explain physical phenomena behind them and to relate these components to brush and commutator wear mechanisms. Finally, conclusions and contributions made by the paper are summarised.

\section{EXPERIMENTAL DATA AND DISCUSSION}

Instrumentation of two industrial DC machines installed at the facility included a large number of sensors installed internally and externally to the motors. A specific feature of this instrumentation is the installation of Hall Effect Flux sensors inside the machines, as shown in Figure 1 for one of the test motors. These sensors allow for direct measurement of flux densities at different points in the main air gap. For the purposes of the analysis presented in this paper, the measurements of flux densities for one of the test machines, under the central point of a main pole and under the central point of an interpole have been selected. These signals have been complemented by the armature current, armature voltage and field current measured at the terminals of the machine. A fragment of experimental data recorded at a rotational velocity of $243 \mathrm{RPM}$, average load of $1000 \mathrm{~A}$ and with a sampling rate of $25 \mathrm{KHz}$ is shown in Figure 2.

\section{A. Armature voltage}

With a six pulse silicon controlled rectifier (SCR), the armature voltage waveform over a period of $50 \mathrm{~Hz}$ consists of 6 fragments of a $50 \mathrm{~Hz}$ sine wave, each given by:

$$
v_{a}(t)=V_{m} \sin \left(\omega_{s} t+\frac{\pi}{3}+\alpha\right), \quad 0 \leq t \leq T_{300}
$$

where $V_{m}$ is amplitude of the input AC line-to-line voltage to the drive; $\omega_{s}=2 \pi \times 50$ is the synchronous frequency for a $50 \mathrm{~Hz}$ supply; $T_{300}$ is the period of the 6 -th harmonic of the supply frequency $(300 \mathrm{~Hz})$; and $\alpha$ is firing angle. The firing angle is defined as the phase angle of firing the thyristor relative to the phase angle that is naturally maintained for an uncontrolled (diode) rectifier (see, for example, [2]). It is typical for thyristor rectifiers to work within a range of firing angles $\frac{\pi}{6}$ to $\frac{5 \pi}{6}$. Then the waveform given by (1) corresponds to an almost straight line fragment of the sine wave, and the 
armature voltage has a saw-tooth shape, as can be seen in Figure 2b).

A linear approximation of (1) shifted to a symmetrical interval $-\frac{T_{300}}{2} \leq t \leq \frac{T_{300}}{2}$ is given by:

$$
v_{a}(t)=V_{D C}-2 V_{m} \frac{t}{T_{300}}
$$

with a corresponding Fourier series:

$$
v_{a}(t)=V_{D C}+\frac{2 V_{m}}{\pi} \sum_{k=1}^{\infty}(-1)^{k} \frac{1}{k} \sin \frac{2 \pi k t}{T_{300}}
$$

where $V_{D C}$ is average (DC) armature voltage. According to (3), it is expected that the spectrum of the armature voltage will contain all harmonics of $300 \mathrm{~Hz}$ with magnitudes decreasing in proportion to $\frac{1}{k}$.

\section{B. Armature current}

The armature current shown in Figure 2a) can be found by using the armature voltage expression (1) and solving the DC motor dynamic equation:

$$
R_{a} i_{a}(t)+L_{a} \frac{d i_{a}(t)}{d t}+E=v_{a}(t)
$$

where $i_{a}(t)$ is armature current; $R_{a}$ and $L_{a}$ are armature resistance and inductance respectively; $E$ is back emf. It can be shown (see, for example, [5]) that the solution of the equation (4) for a thyristor-based drive is given by:

$$
\begin{aligned}
i_{a}(t) & =\frac{V_{m}}{\left|Z_{a}\right|}\left\{\sin \left(\omega_{s} t+\frac{\pi}{3}+\alpha-\beta\right)-\sin \left(\frac{\pi}{3}+\alpha-\beta\right) \times\right. \\
& \left.\times e^{-\frac{t}{T_{a}}}\right\}-\frac{E}{R_{a}}\left(1-e^{-\frac{t}{T_{a}}}\right)+i_{a i} e^{-\frac{t}{T_{a}}}
\end{aligned}
$$

where $\left|Z_{a}\right|=\sqrt{R_{a}^{2}+\left(L_{a} \omega_{s}\right)^{2}}$ is the machine impedance magnitude; $\beta=\tan ^{-1} \frac{L_{a} \omega_{s}}{R_{a}}$ is the machine impedance angle; $T_{a}=\frac{L_{a}}{R_{a}}$ is the armature time constant; and $i_{a i}=i_{a}(t=0)$ is the initial current at the beginning of each cycle. An approximate expression for the armature current can be found by integrating the linear armature voltage approximation (2), conveniently shifted to the interval $0 \leq t \leq T_{300}$, as:

$$
i_{a}(t)=i_{a i}+\frac{V_{m}}{L_{a}} t\left(1-\frac{t}{T_{300}}\right)
$$

The corresponding Fourier series is given by:

$$
i_{a}(t)=i_{a i}+\frac{V_{m} T_{300}}{6 L_{a}}-\frac{V_{m} T_{300}}{L_{a} \pi^{2}} \sum_{k=1}^{\infty} \frac{1}{k^{2}} \cos \frac{2 \pi k t}{T_{300}}
$$

It is therefore expected that the spectrum of the armature current waveform shown in Figure 2a) will also contain all harmonics of $300 \mathrm{~Hz}$ but their magnitudes will decay quickly with the harmonic number in proportion to $\frac{1}{k^{2}}$. This is due to a smoothing effect of the armature inductance.

It can also be appreciated from Figure 2a) that the pulses in the current waveform are slightly out of balance: every second pulse is lower than the adjacent pulses. This can be attributed to an assymetry in the thyristor circuit, i.e. between positive and negative rails in the rectifier. Another likely reason for the observed unbalance is a very limited current control bandwidth, possibly resulting in fluctuations of the current around its actual set point repeating every second control cycle (note that the control frequency in the six pulse SCR drive is $300 \mathrm{~Hz}$ ). The unbalance can be accounted for by adding to the main waveform given by (6) a symmetrical square wave of $150 \mathrm{~Hz}$ with the Fourier series given by:

$$
i_{s q}(t)=\frac{4 I_{s q}}{\pi} \sum_{k=0}^{\infty}(-1)^{k} \frac{1}{2 k+1} \sin \frac{2 \pi(2 k+1) t}{T_{150}}
$$

where $I_{s q}$ is amplitude of the square wave (approximately $4.5 \%$ of the average armature current or $10 \%$ of the $300 \mathrm{~Hz}$ harmonic amplitude); $T_{150}=2 T_{300}$ is period of $150 \mathrm{~Hz}$. According to (8), the magnitude unbalance of the pulses will result in presence in the armature current spectrum of odd harmonics of $150 \mathrm{~Hz}$ with magnitudes decreasing in proportion to $\frac{1}{2 k+1}$.

\section{Field current}

The field current waveform shown in Figure 2e) also looks like an output current of a 6-pulse rectifier. Due to a high value of field inductance, $300 \mathrm{~Hz}$ pulsations (with an amplitude of approximately $2 \%$ of the average field current value) are much smaller than in the armature current waveform. The $150 \mathrm{~Hz}$ unbalance is also present in Figure 2e) and has the same order of magnitude as in the armature current waveform (approximately $4 \%$ of the average current value). Similar to the spectrum of the armature current, the spectrum of the field current will contain harmonics of $300 \mathrm{~Hz}$, as well as odd harmonics of $150 \mathrm{~Hz}$ according to (8).

\section{Main pole flux density}

Neglecting reluctance of a magnetic part of the flux lines path, the main pole flux density can be expressed as:

$$
B_{m p}=S\left(\frac{N_{m p} i_{f} \mu_{0}}{l_{m g}}\right)=S\left(C_{m p} i_{f} \Lambda_{m g}\right)
$$

where $S(\cdot)$ is non-linear (saturation) characteristic of the main field; $N_{m p}$ is the main pole turns number; $i_{f}$ is main field current; $\mu_{0}$ is permeability of free space; $l_{m g}$ is the length of the main pole air gap; $\Lambda_{m g}=\frac{\mu_{0} A_{m p}}{l_{m g}}$ is magnetic permeance of the main pole air gap; $A_{m p}$ is the main pole cross-sectional area; and $C_{m p}=\frac{N_{m p}}{A_{m p}}$ is a constant.

According to (9), the main pole flux density can change either due to the main field current $i_{f}$ or due to the permeance of the main pole air gap $\Lambda_{m g}$. As the field current varies no more than $\pm 4 \%$ of its average value, high amplitude variations of the main pole flux density observed in Figure 2d) are predominantly due to the main gap permeance variations (not to be confused with $300 \mathrm{~Hz}$ ). This becomes clear when considering an armature "slot effect": conductors of the armature winding are laid in slots; when a slot passes by a stationary installed main pole flux sensor, the effective air gap increases 
and its permeance drops. Frequency of the "slot effect" is given by:

$$
f_{s l}=n_{r p m} \frac{N_{s l}}{60}
$$

where $n_{r p m}$ is rotational velocity in RPM; and $N_{s l}$ is the number of slots of the armature winding.

It can be assumed, to a first approximation, that the main air gap magnetic permeance $\Lambda_{m g}(t)$ is some (close to a rectangular) waveform with Fourier series containing the $f_{s l}$ component and its harmonics:

$$
\Lambda_{m g}(t)=\Lambda_{0}+\sum_{k=1}^{\infty} \Lambda_{k} \cos \left(2 \pi k f_{s l} t+\varphi_{\Lambda k}\right)
$$

where $\varphi_{\Lambda k}$ is the phase of the $k$-th harmonic, which can take any value for a general non-symmetrical rectangular waveform. In other words, both sine and cosine terms may be present for each frequency. Then the following harmonics will appear in the $B_{m p}$ spectrum (in descending order of magnitude):

- the slot effect component $f_{s l}$ and its harmonics, as in the expression for permeance (10);

- $150 \mathrm{~Hz}$ and $300 \mathrm{~Hz}$ harmonics as in the field current;

- beat frequencies between the two groups, in particular, $\left(f_{s l} \pm 150\right) \mathrm{Hz}$ and $\left(f_{s l} \pm 300\right) \mathrm{Hz}$.

\section{E. Interpole flux density}

The interpole flux density can be expressed in a similar way to (11), except for saturation, due to the following reasons:

- the interpole itself creates only a small field to compensate for armature reaction and assist commutation;

- the interpole air gap is longer than the main pole air gap, hence higher reluctance;

- saturation of the interpole by the flux lines of the adjacent main poles is prevented from by using non-magnetic indents (shims) at the root of the interpole pole piece.

Therefore, the interpole flux density can be expressed as:

$$
B_{i p}=\frac{N_{i p} i_{a} \mu_{0}}{l_{i g}}=C_{i p} i_{a} \Lambda_{i g}
$$

where $N_{i p}$ is the interpole turns number; $l_{i g}$ is interpole air gap; $\Lambda_{i g}=\frac{\mu_{0} A_{i p}}{l_{i g}}$ is magnetic permeance of the interpole air gap; $A_{i p}$ is the interpole cross-sectional area; and $C_{i p}=\frac{N_{i p}}{A_{i p}}$ is a constant.

The "slot effect" that explained variations in the main pole flux density is also relevant to the interpole flux density. Magnetic permeance of the interpole air gap $\Lambda_{i p}(t)$ can be expressed in a form similar to (10). However, the amplitudes $\Lambda_{i k}$ of its harmonics will be much lower relative to the mean value $\Lambda_{i 0}$ due to the fact that the interpole air gap is longer, and its relative change due the armature slots is much smaller. Variations seen in the interpole flux density waveform of Figure IIc) are clearly dominated by $300 \mathrm{~Hz}$ ripple of the armature current origin. The following harmonics will appear in the $B_{i p}$ spectrum (in descending order of magnitude):

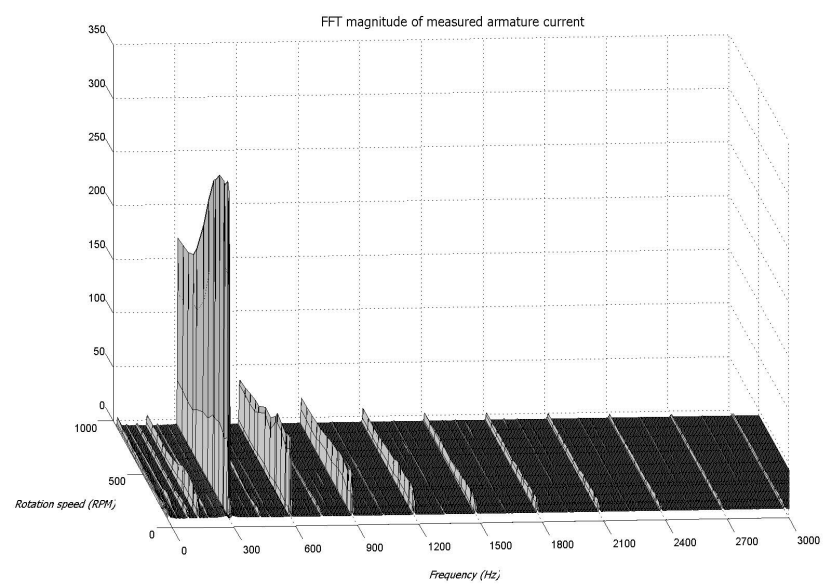

Figure 3. FFT magnitude of the armature current at different speed

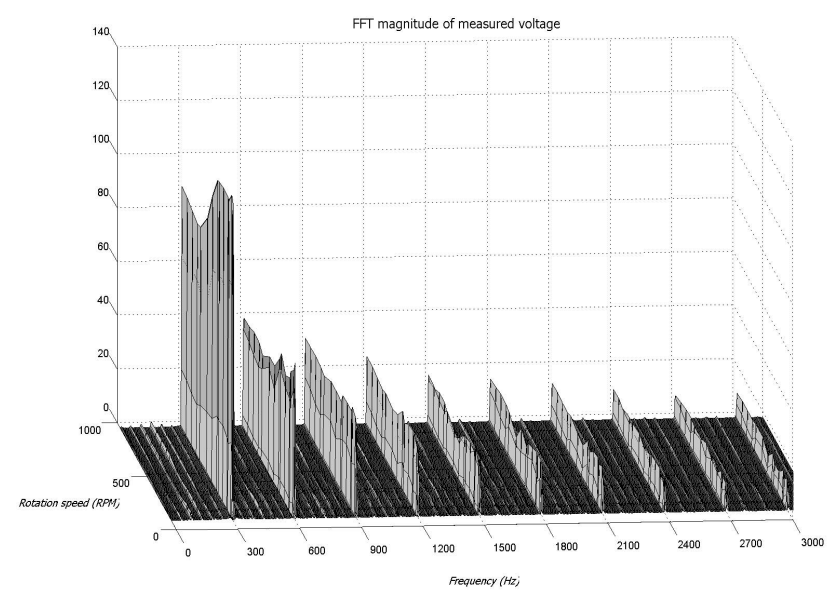

Figure 4. FFT magnitude of the armature voltage at different speed

- $300 \mathrm{~Hz}$ and its first few harmonics as in the armature current;

- the slot effect component $f_{s l}$ and its harmonics, as in the interpole air gap permeance;

- $150 \mathrm{~Hz}$ and its odd harmonics as in the armature current;

- cross-harmonics between the above groups, in particular, $\left(f_{s l} \pm 300\right) \mathrm{Hz},\left(f_{s l} \pm 600\right) \mathrm{Hz}$ and $\left(f_{s l} \pm 150\right) \mathrm{Hz}$.

\section{Methods AND Results of Data Analysis}

\section{A. Methods of analysis}

In the previous section we achieved some theoretical understanding of the expected spectra of the main measurable signals. Now we will calculate spectral characteristics of the experimentally measured waveforms based on Fourier analysis. The following methods within Fourier analysis family will be used:

- Magnitude of the Fast Fourier Transform (FFT) for each signal - this will allow us to compare the theoretically predicted and the experimentally developed spectra;

- Magnitude of frequency response for related signal pairs, for example, interpole flux density to armature current - 


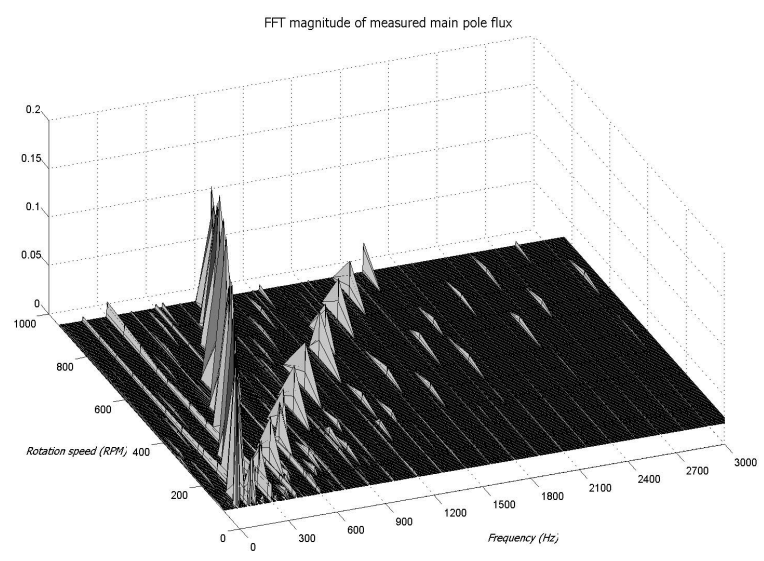

Figure 5. FFT magnitude of the main pole flux at different speed

this will show how one signal is transformed to result in the other; spectral component that are gained via this transformation will be emphasized;

- Power spectral density (PSD) for some signals of interest - this will be particularly important to reliably identify, with good resolution and accuracy, low frequency components in signals related to commutation.

Experimental data was collected in steady state at 14 different rotational velocities from 85 RPM to 947 RPM, which covered the full range of the motor operation speeds. For all the data the set point for the armature and field currents were the same: $1000 \mathrm{~A}$ and $120 \mathrm{~A}$ respectively. However, at rotation velocities above 400 RPM both armature and field current started dropping and eventually reached each about a half of its set point value. As the harmonics content of the signals of interest is not proportional to their mean values, no data normalisation was done, apart from detrending the data by removing the DC offset.

Comparison of the spectra at different rotational velocities will allow us to observe how the harmonic content of the signals evolves with changing of the operation speed, and to distinguish between speed-dependent and speed-independent frequency components. This comparison will be done by presenting the spectra of each signal:

- by a 3D plot, with frequency and rotational velocity as the two independent coordinates;

- by combining spectra for all rotational velocities in one plot as functions of frequency with linear scale applied (this will highlight spectral components independent of the rotational velocity);

- as above but as functions of frequency normalised to the frequency of rotation (this will highlight spectral components related to the rotational velocity).

Each of the above mentioned 14 data series included 90112 points of sampled at $25 \mathrm{KHz}$ of the five signals of interest (the same signals that appear in Figure 2). To achieve a balance between accuracy of spectral estimations and their resolution, the FFT calculations will be based on 16384 points, windowed

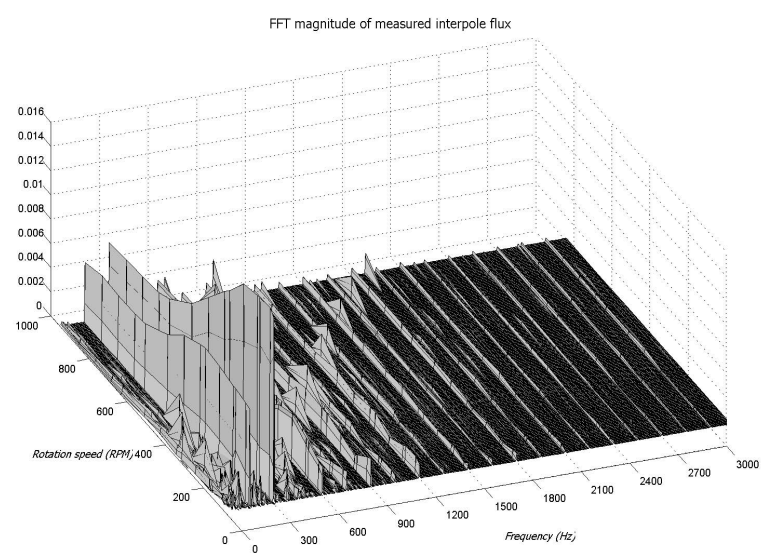

Figure 6. FFT magnitude of the interpole flux at different speed

by Hamming window (see [9]) of the corresponding size, with $50 \%$ overlap. The calculated FFT magnitude is given by:

$$
|F(k)|=\frac{2 T_{s}}{M} \sum_{m=1}^{M} \frac{1}{N}\left|\sum_{l=1}^{N} w(l) x^{(m)}(l) e^{-j \frac{2 \pi}{N} k(l-1)}\right|
$$

where $T_{s}=40 \cdot 10^{-6} \mathrm{sec}$ is the sample time; $M=10$ is the number of segments; $N=16384$ is the segment data length; $w(l)$ is the window function (Hamming in this case); $x^{(m)}(l)$ is the $m$-th overlapped sampled data segment.

Magnitude of the frequency response between two signals was found by dividing the respective FFT magnitudes calculated according to (12). To achieve a higher resolution in PSD calculations, an increased segment size of 65536 points was used with $31.25 \%$ overlap between the windows. PSD was calculated based on standard modified Welch periodogram (see [9]) as:

$$
P_{x x}(k)=\frac{2 T_{s}}{M} \sum_{m=1}^{M} \frac{1}{N}\left|\sum_{l=1}^{N} w(l) x^{(m)}(l) e^{-j \frac{2 \pi}{N} k(l-1)}\right|^{2}
$$

where $M=3$ is the number of segments; $N=65536$ is the segment data length and $w(l)$ is the Hanning window function.

\section{B. Results and discussion}

1) FFT magnitudes: FFT magnitudes for the armature current, armature voltage, main pole flux density, interpole flux density and field current are shown in Figure 3, Figure 4, Figure 5, Figure 6 and Figure 7, respectively. Spectra of Figures 3-7 include the rotational velocity as the second argument to show evolutions of the signal frequency content with the velocity of rotation.

From examining the waveforms of Figure 2, spectra of Figures 3-7 and coupling these with the theoretical predictions of the previous section, the following observations can be made about each signal of interest:

- Armature voltage. As predicted, the armature voltage spectrum of Figure 4 is rich in harmonics of $300 \mathrm{~Hz}$ that 


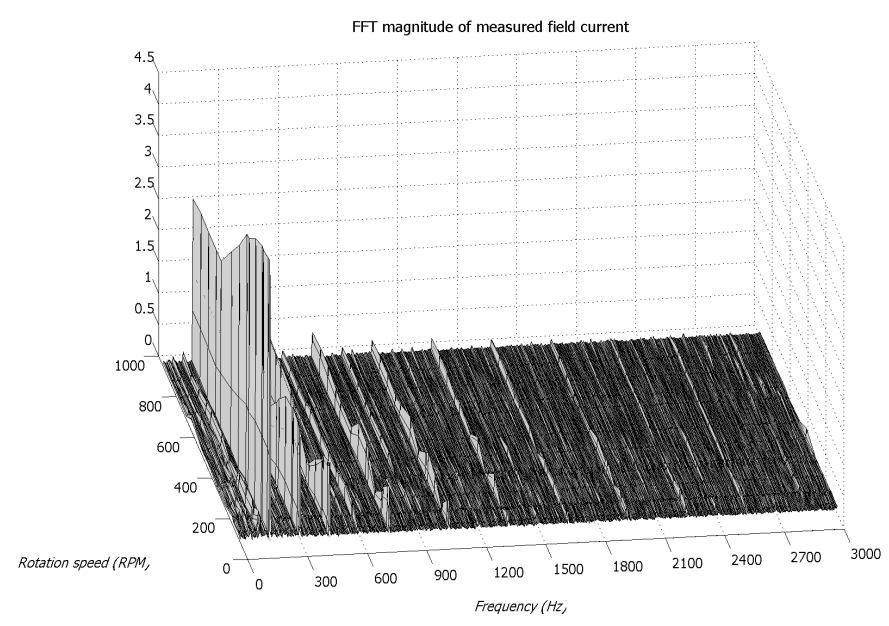

Figure 7. FFT magnitude of the field current at different speed

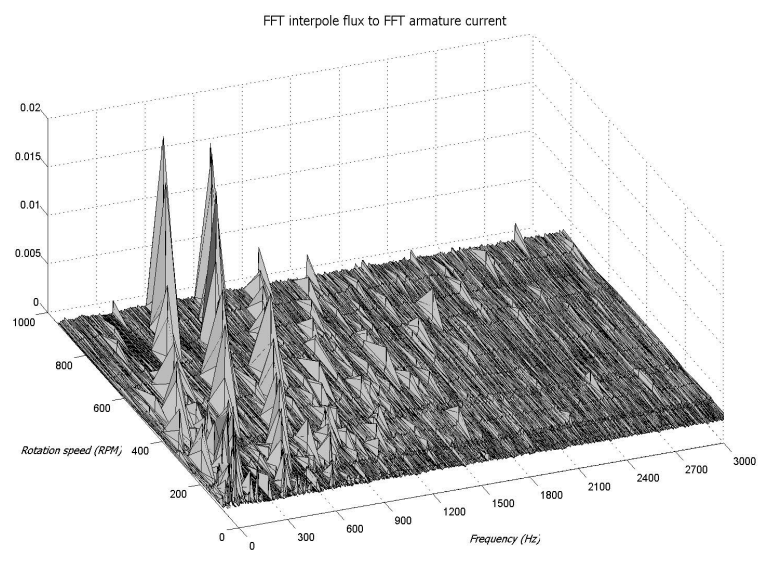

Figure 8. Magnitude of frequency response of interpole flux density to armature current at different speeds

decay slowly with the harmonic number. Apart from that, a large number of $50 \mathrm{~Hz}$ harmonics are present in the spectrum. A voltage spike followed by a damped high frequency oscillation seen in the waveform of Figure $2 b$ ) deserves a particular interest. Its position relative to the beginning of a $300 \mathrm{~Hz}$ cycle is consistent for a given rotational velocity, which suggests that it originates from the thyristor firing circuit.

- Armature current. As predicted, the armature current spectrum of Figure 3 includes the $300 \mathrm{~Hz}$ component and its harmonics decay fast with the harmonic number. The $150 \mathrm{~Hz}$ component and its odd harmonics are also pronounced, as well as low order $50 \mathrm{~Hz}$ harmonics. A low frequency component present at all rotational velocities needs additional signal processing for a better resolution. Another interesting observation from Figure 2a) is that every second current pulse is not only higher but also consistentently noisier than the adjacent pulses. The same is true for the interpole flux density waveform of Figure 2c) and needs a further investigation.

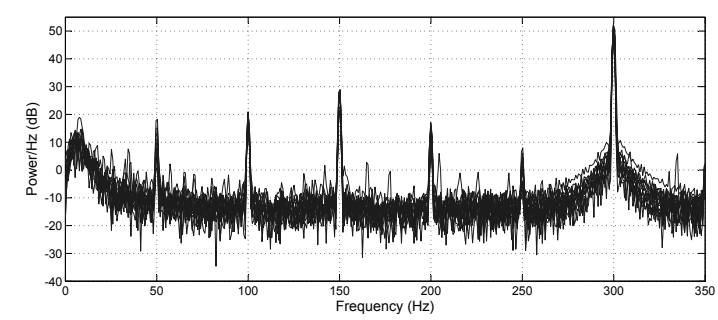

Figure 9. PSD for the armature current (zoomed)

- Main pole flux density. Its spectrum shown in Figure 5 is dominated by the "slot effect" component and its second harmonic. The third and the fourth harmonics of $f_{s l}$ can also be seen. Spectral components of $150 \mathrm{~Hz}, 300 \mathrm{~Hz}$ and $\left(f_{s l} \pm 300\right) \mathrm{Hz}$ are appreciable.

- Interpole flux density. The measurements of the interpole flux density are taken in the middle of the commutation zone and are particularly important for commutation diagnostics. The interpole flux spectrum shown in Figure 6 contains strong $300 \mathrm{~Hz}$ and $150 \mathrm{~Hz}$ components and their harmonics (originating from the armature current), as well as the "slot effect" component.

- Field current. The strongest component in field current spectrum shown in Figure 7 is $150 \mathrm{~Hz}$. Harmonics of $300 \mathrm{~Hz}$ and odd harmonics of $150 \mathrm{~Hz}$ are also present. Evolution of the $150 \mathrm{~Hz}$ component magnitude with rotational velocity is primarily due to field weakening taking place at higher velocities.

2) Frequency response: The magnitude of the frequency response of the interpole flux density to the armature current is shown in Figure 8 as a 3D plot. It helps to highlight the frequency components not so well pronounced in the FFT magnitude of Figure 6, i.e. beat frequencies between the SCR-drive related and the slot effect related components. The second row of peaks in Figure 8 corresponds to $f_{s l}$, and to to the right from it are $\left(f_{s l}+300 k\right)$ components. The first row of peaks in Figure 8 corresponds to $\left(f_{s l}-300\right) \mathrm{Hz}$, and to left from a few small peaks at $\left(f_{s l}-600\right) \mathrm{Hz}$ are just noticeable.

Another meaningful frequency response function, namely, of the main pole flux to the field current, showed no additional information to that present in the plot for the main pole flux, and is not included in this paper.

3) Power spectral density: As mentioned earlier, some of the signal spectra needed a better resolution in the low frequency range. This is addressed in Figure 9 and Figure 10 and Figure 10 that show PSDs (for all 14 data series in one plot) for the armature current and for the interpole flux density with different axes scaling. The axis in Figure 9 is the absolute frequency $(\mathrm{Hz})$ and helps to reveal the presence in the armature current of $50 \mathrm{~Hz}$ and its harmonics. The corresponding plot with normalised frequency was not very informative and is not included in the paper.

In Figure 10 the interpole flux density is presented versus the normalised frequency (referred to the frequency of rotation). It clearly shows the presence of the fundamental (rotation) frequency and its harmonics with numbers $6 k$ and 


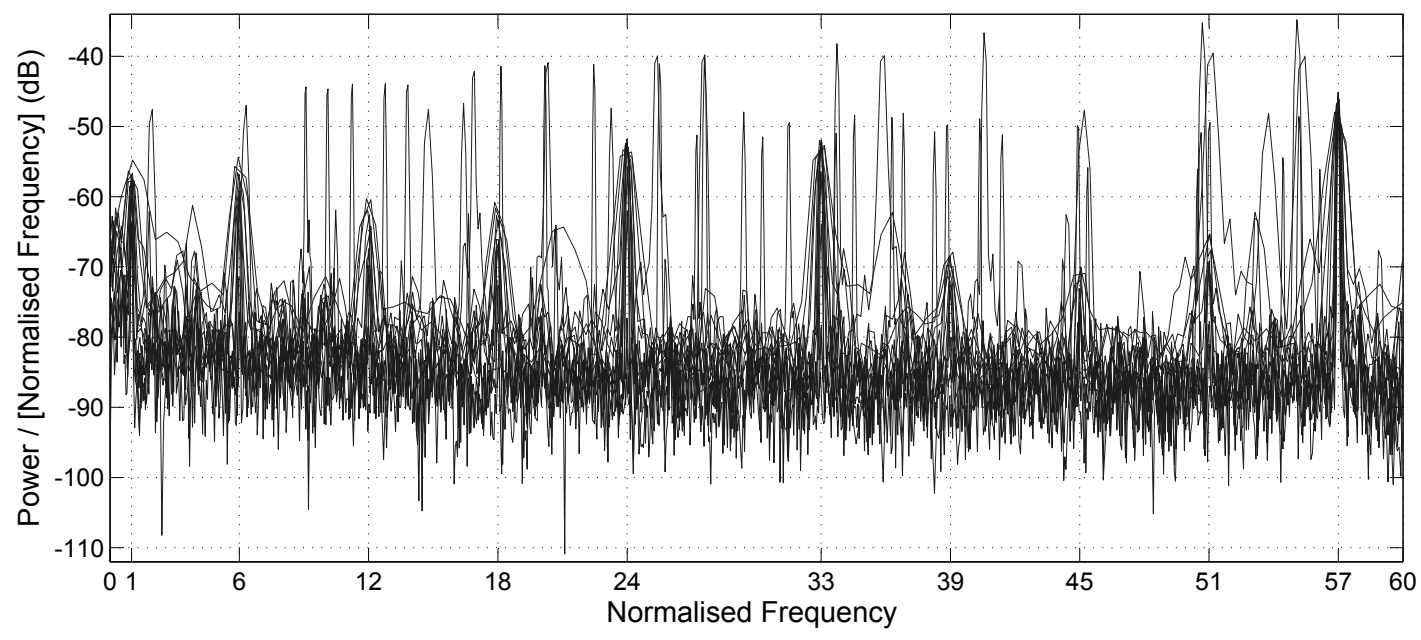

Figure 10. PSD for the interpole flux density (zoomed)

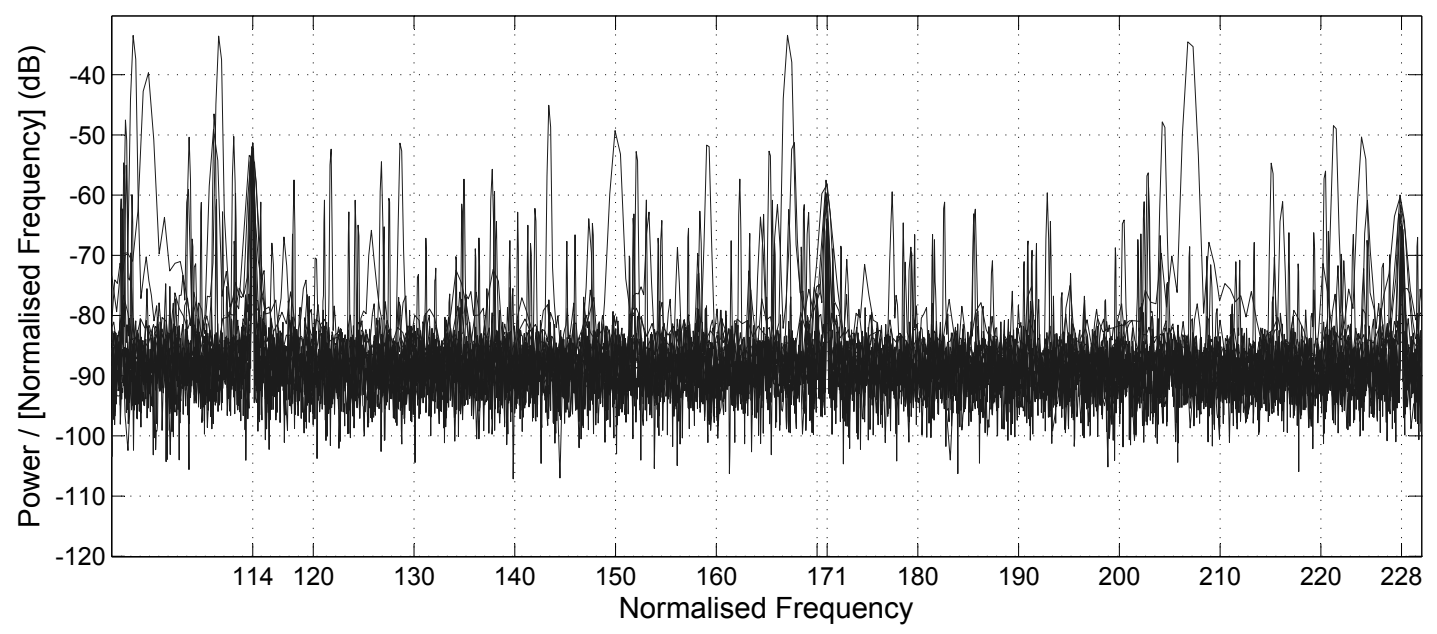

Figure 11. PSD for the interpole flux density (continued)

$(57-6 k)$. Note that 6 is the number of poles of the machine, and 57 is the number of the armature slots. According to [4], presence of such harmonics may be related to the armature dynamic eccentricity. Examination of the interpole flux density plot further along the frequency axis (see Figure 11) also revealed consistent presence of the 2-nd, 3-rd and 4-th harmonics of the slot frequency, which correspond to the 114-th, 171-st and 228-th harmonics of the frequency of rotation. Frequency of commutation for the machine under question is 3 times the slot frequency. Problems with commutation will manifest themselves in raising of the 171-st harmonic of the rotation frequency while other harmonics (number 57, 114 and 228) associated with the slot effect will not raise.

When conducting the experiments it was noticed that at some rotational velocities between 250 and $360 \mathrm{rpm}$ the mechanical structure of the facility experienced low frequency vibrations. Low frequency range PSDs for the interpole flux densities at 250, 327 and $355 \mathrm{rpm}$ (corresponding to slot frequencies 238, 310.5 and $337 \mathrm{~Hz}$, respectively) are shown in Figure 12. Components between 4 and $6 \mathrm{~Hz}$ correspond to the frequencies of rotation. Components of 10.5, 37 and $62 \mathrm{~Hz}$ correspond to $(310.5-300) \mathrm{Hz},(337-300) \mathrm{Hz}$ and $(300-238) \mathrm{Hz}$, respectively, i.e. to the beat frequencies between the slot effect and $300 \mathrm{~Hz}$ from the SCR drive. One can appreciate from Figure 12 that these beat frequency components are almost of the same magnitude as the slot effect frequency components.

It is reasonable to suggest a beat frequency initiates a small vibration (e.g. by a magnetostriction effect), which becomes significantly amplified if coincides with a frequency of mechanical resonance. Such vibrations transferred to the brushes relative to the commutator surface may lead to an increased rate of wear of both (see [3]). Vibration sensors are currently being installed on the brush arms and the motor case to check the vibration hypothesis. 


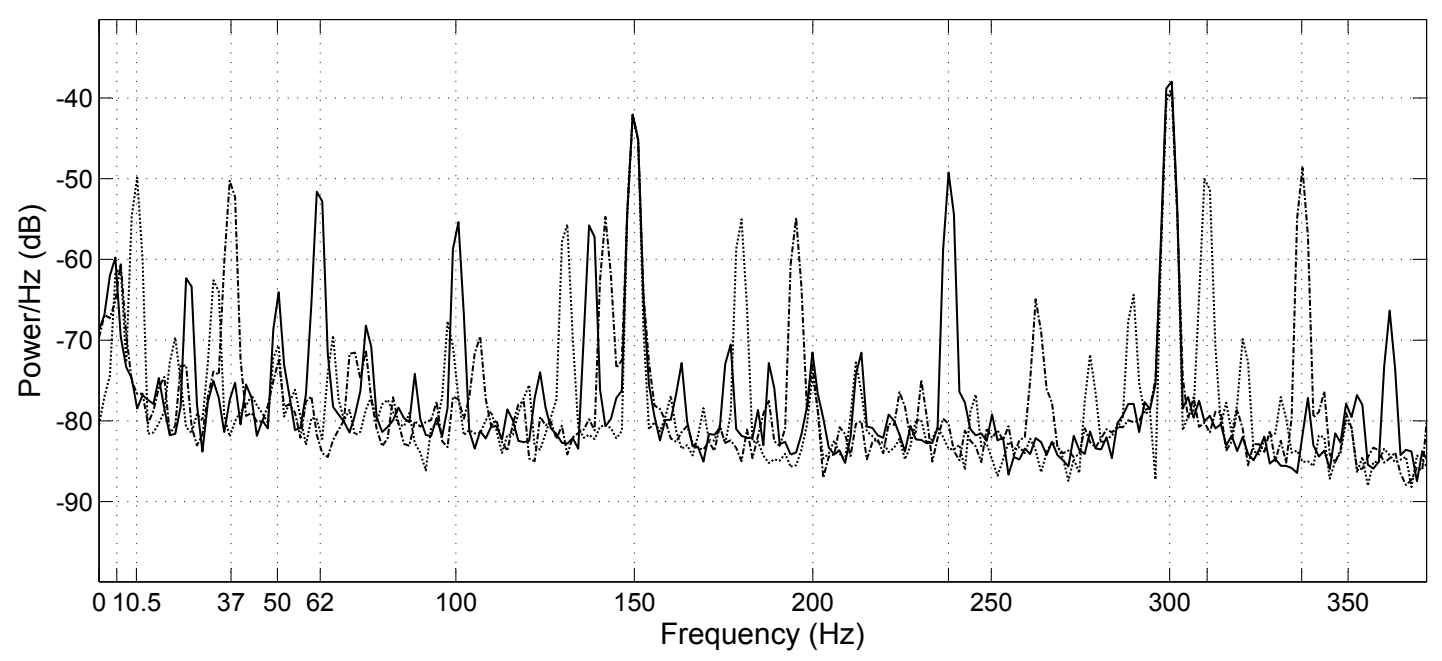

Figure 12. PSD for the interpole flux density for 250, 327 and 355 RPM

\section{CONCLUSIONS AND CONTRIBUTIONS}

The paper presented a detailed theoretical analysis of the critical external and internal signals of the thyristor-fed DC motor used in mining applications. It presented experimentally obtained waveforms and their calculated spectra corresponding to different rotational velocities. Important observations were made that are relevant to condition monitoring of the commutation group and the motor mechanical condition, such as:

- presence of spikes in the armature voltage waveform;

- a pattern of unbalance and noise in the armature current;

- presence of $6 k$ harmonics of the rotation frequency, as well as beat frequencies between these and the slot frequency, in the interpole flux density, which are indicative of the mechanical eccentricity;

- presence of harmonics of the slot effect frequency (including the frequency of commutation) in the interpole flux density, which can be used to distinguish commutation deterioration;

- low frequency range components in the interpole flux density, which can create conditions for mechanical resonances.

The main contribution of this paper is the proposed strategy for condition monitoring of DC machines. The main principles of condition monitoring of rotating machines are well known (see, for example [4]). When applying these to DC machines it is usually suggested to monitor frequency components in the armature current ( [8]). However, when a DC machine is fed from a SCR drive, $300 \mathrm{~Hz}$ and associated harmonic components tend to mask other features. In fact, no interesting harmonic components were found at all in the examined armature current spectra for the test DC motor. However, the flux density measurements taken under the interpole offered a large number of consistent and informative frequency components in its spectra.

It is therefore proposed in this paper to monitor the armature current for the SCR drive associated issues and to monitor the interpole flux density for the range of other issues including:

- commutation defects;

- mechanical unbalance and eccentricity;

- mechanical resonances;

and potentially other issues. The next stage of the experiments will cyclic load testing to simulate the digging conditions, verify the hypotheses and develop correlation models between certain conditions and measurable signals.

\section{REFERENCES}

[1] R.E. Betz, G. Mirzaeva, , and T.J. Summers. A dynamic dynamometer for testing of mining dc motors. In Accepted for presentation at $45 \mathrm{th}$ IEEE Industry Applications Society Annual Meeting, Houston, TX, USA, 3-7 October 2010.

[2] B.R.Pelly. Thyristor controlled converters and cycloconverters: operation, control, and performance. Wiley-Interscience, New York, 1971.

[3] F. Flinders, J. Zhang, and W. Oghanna. Investigation on excessive commutator and brush wear in dc traction motors. In IEEE International Conference on Power Electronics and Drive Systems, 1999. PEDS 1999 Conference Record of the, volume 1, pages 200-205, 27-29 July 1999.

[4] M. Hajiaghajani, H.A. Toliyat, and I.M.S. Panahi. Advanced fault diagnosis of a dc motor. IEEE Transactions on Energy Conversions, 19(1):60-65, 2004.

[5] R. Krishnan. Electric motor drives: modeling, analysis and control. Prentice-Hall, Upper Saddle River, New Jersey 07458, 2001.

[6] G. Mirzaeva, R.E. Betz, and T.J. Summers. Evaluation of current density in dc motor brushes for mining machines based on air gap field measurement. In Industry Applications Conference, 2009. 44th IAS Annual Meeting. Conference Record of the, pages 1-8, 4-8 Oct 2009.

[7] G. Mirzaeva, R.E. Betz, T.J. Summers, and I. Marxsen. Development of a unique dc motor test facility in the hunter valley. In Proc. of the Australian Mining Technology Conference: Smart Technologies for Sustaining the Minerals Boom. 16-18 September 2008, Twin Waters, QLD, Australia, pages 95-106, 2008.

[8] S. Nandi, H.A. Toliyat, and Xiaodong Li. Condition monitoring and fault diagnosis of electrical motors - a review. IEEE Transactions on Energy Conversions, 20(4):719-729, 2005.

[9] A.V. Oppenheim and R.W. Schafer. Discrete-Time Signal Processing. Prentice-Hall, 1989. 